\title{
The Effect of Microblog's Content on Customer Satisfaction
}

\author{
Hanxiaoni*, Xuwenti \\ School of Management and Economics, Beihang University, Beijing, China
}

\section{Email address:}

hanxiaoni11@163.com (Hanxiaoni),xuwenti@buaa.edu.cn (Xuwenti)

\section{To cite this article:}

Hanxiaoni, Xuwenti. The Effect of Microblog's Content on Customer Satisfaction. International Journal of Economics, Finance and Management Sciences. Vol. 3, No. 5, 2015, pp. 611-615. doi: 10.11648/j.ijefm.20150305.34

\begin{abstract}
The new media marketing has show out a great potential with the rapid development of new media in recent years. As an important kind of new media, microblog has become a good platform for communication and interaction between enterprises and customers. The Xiaomi mobile phone is as an example to investigate the influence of enterprise microblog marketing on customer satisfaction. The attitudes of customers on different enterprise microblog contents are examined by combining experiment and questionnaire survey method. The results show that there is significant difference between the impact of social interaction microblog and task-oriented interaction on customer attitude. Social content is more likely to raise the attention of customers up. However, customers tend to support the product after they read task-oriented contents. Microblog has a significant impact on customer satisfaction, which should be used to build good customer relationships.
\end{abstract}

Keywords: Microblog Marketing, Social Content, Task-Oriented Content, Customer Satisfaction

\section{微博互动内容对顾客满意度的影响研究}

\section{韩晓妮 ${ }^{*}$, 许文媞}

经济管理学院, 北京航空航天大学, 北京, 中国

\section{邮箱}

hanxiaoni11@163.com(韩晓妮), xuwenti@buaa. edu. cn (许文媞)

摘要: 随着近年来新媒体的迅猛发展, 利用新媒体平台开展营销显现出巨大的潜力。作为一种重要的新媒体, 微博因 为其互动性、沟通性和广泛性, 成为企业与顾客交流互动的良好平台。本文以小米手机为例, 采用实验和问卷调查相 结合的方法, 探讨顾客对企业不同微博内容的态度, 进而考察企业微博营销对顾客满意度的影响。经过研究发现, 社 会性微博互动内容与任务导向型微博互动内容, 对顾客态度和购买意愿影响不同。社会性互动内容更容易引起被试者 的微博参与意愿（点赞、评论或者转发）, 但任务导向型互动内容对顾客购买意愿影响更大。被试者对企业微博的态 度, 与对企业的满意度显著相关，企业应该利用好微博平台，建立良好顾客关系。

关键词：微博营销, 社会性互动, 任务导向型互动, 顾客满意度

\section{1. 引言}

20 世纪互联网的诞生与发展改变了人们的生活习惯和 消费观念，越来越多的人利用网络空间来开展社会活动和 消费活动。近年来新媒体的兴起, 为人们更加自由的表达 观点抒发情绪提供了便捷的平台，同时显示出强大的营销 功能和潜在的商业价值。与传统只强调覆盖率的单向式营
销不同, 新媒体营销是指利用新媒体平台, 如博客、微信 等进行营销的模式, 具有体验性、沟通性、差异性、创造 性、关联性等特点。通过新媒体平台, 企业可以以更低的 成本, 更大的范围搜索消费者, 利用网络和舆论领袖的声 音更好地向消费者传达企业信息，扩大企业影响力。

微博 (Microblog) 是微型博客的简称, 是一个基于用 户关系的信息分享、传播以及获取平台。用户可以在微博 
上发表140 字以内的文字、图片、链接等, 并设有转发、 评论、点赞等功能, 来实现用户与微博传播平台之间的信 息沟通与交流，以及微博用户之间的信息交换与人际交往。 截止至2014年6月, 中国微博用户数量达到2.75亿, 网民 使用率为 $43.6 \%$, 受众广泛、影响力大, 使微博展现出巨 大的商业潜力。企业微博与其他官方沟通平台最大的差别 就在于企业可以通过消费者的转发和评论行为注意到消 费者对企业的心理感受。如果企业能够因势利导, 对消费 者的心声作出恰当的回应, 就能增强粉丝对企业的认同感, 并提升企业和粉丝的关系。

目前, 许多企业纷纷开通微博, 旨在更好地宣传企业 和产品, 但是一味的发布产品信息, 结果往往适得其反。 企业利用微博平台, 应该从粉丝角度出发, 通过科学地发 布微博内容, 才能建立良好顾客关系, 扩大企业影响力, 提升品牌价值。企业发布的微博内容, 以及发表微博的频 率等, 都应该从顾客角度出发, 这是值得企业探讨的问题。 本文以小米手机为例, 从顾客角度出发, 利用专业眼动设 备, 采用实验和问卷调查相结合的方法, 探讨顾客对企业 不同微博内容的态度, 进而考察企业微博营销对顾客满意 度和购买意愿的影响。通过研究, 为企业科学开展微博营 销提供实证依据, 并根据结果提出建议, 达到提升企业形 象, 提高顾客忠诚度的目的。

\section{2. 研究方法}

\section{1 . 实验样本}

样本来源于北京某高校的在校大学生和研究生, 共 28 名被试者, 其中男性13名, 女性15名, 年龄集中于18到35 岁。所有被试者均视力正常（包括矫正后的）, 之前未参 与过本研究。

\section{2. 实验仪器}

实验仪器为瑞典生产的Tobi i T120眼动仪, 采样频率 $120 \mathrm{~Hz}$, 显示屏大小为 19 英寸液晶显示屏, 32 位增强色, 分辨率为 $1024 \times 768$ 像素, 刷新率为 $45 \mathrm{~Hz}$ 。是一种记录人 眼动轨迹变化的精密仪器。它能捕捉并记录下诸如注视点、 注视时间、视觉轨迹等人眼的任何一个细小动作并提取相 关数据。被试者与显示屏幕的距离约为 $60 \mathrm{~cm}$, 眼睛正对 显示器的中心。被试者眼睛的注视情况通过两个微型摄像 机输入微机, 数据采样率为每秒 1000 次, 相邻两次采样的 间隔为 $1 \mathrm{~ms}$ 。

\section{3. 实验材料}

在理论分析和预调研的基础上，确定实验材料为截取 自小米公司新浪微博的若干张图片, 微博篇幅、图片大小、 界面均保持一致。根据理论分析, 将微博互动内容分为两 类, 社会性互动内容和任务导向型内容。社会性互动内容 的目的是建立和提升双方之间良好的社会关系, 注重满足 对方的社会情感需求, 内容与企业并不直接相关, 主要包 括社会性交流、专业知识提供和情感沟通。任务导向型互 动内容是企业在微博上展示产品、企业形象等与企业密切
相关的信息, 让消费者快速地了解企业和产品, 并激励顾 客与企业共同创造价值, 扩大企业的影响力。主要包括产 品信息发布、企业形象维护和共同创造活动三个方面。

按照微博内容的类型, 将微博图片分为六组, 每组三 张图片, 共从小米公司微博截取十八张图片。按照社会性 互动内容和任务导向型互动内容相互交错的顺序, 相应放 置每组图片。在每组微博图片之后，均设置相同的四个问 题, 来考察消费者对微博内容的态度 (是否愿意点赞、评 论或者转发), 以及对企业的态度（企业形象是否上升、 是否有意愿购买企业产品), 利用五分李克特量表进行调 查 (1表示非常不同意, 2 表示比较不同意, 3 表示一般, 4 表示比较同意, 5 表示非常同意）。微博内容类型及实验 中具体图片顺序如表1所示。

表1 微博内容分类表。

\begin{tabular}{ll}
\hline 图片顺序 & 内容分类 \\
\hline 1. 企业形象宣传 & 任务导向型 \\
2. 手机专业知识 & 社会性 \\
3. 企业产品信息 & 任务导向型 \\
4. 社会性交流 & 社会性 \\
5. 名人娱乐化传播 & 任务导向型 \\
6. 与粉丝情感沟通 & 社会性 \\
\hline
\end{tabular}

\section{4. 实验过程}

在实验中, 被试者首先在电脑上进行眼动实验, 结束 后通过问卷收集个人信息, 由系统交代引导语, 被试者全 程独立完成实验。整个过程中, 实验室保持安静, 被试者 选择一个舒服的坐姿进行实验, 避免在实验中大幅动作, 影响实验数据的准确性。实验完成后, 被试者得到一份礼 物。

\section{5. 数据处理}

针对眼动仪记录数据, 包括注视时长、注视次数等, 运用Tobii Studio软件进行分析。对考察顾客满意度的问 题, 利用SPSS19.0软件进行统计分析。

\section{3. 结果分析}

实验数据分析主要分为两部分, 一部分是通过对被试 者眼动数据的分析, 来考察被试者对不同微博内容的关注 点及关注度; 另一部分是关于每组图片后面问题的分析, 即不同企业微博内容对被试者满意度和购买意愿的影响。

\section{1. 眼动数据分析}

针对收集到 28 份数据, 去掉眼动数据质量为二星以下 的被试者, 得到 27 份有效数据。眼动热点图是根据被试者 汶览图片时的注视时间和注视次数, 来观察被试者的关注 点, 热点图的区域中, 颜色越趋于红色, 表明被试者对该 区域的注视时间和次数越多, 关注度越高。将所有被试者 的注视时间点汇总起来, 选取其中具有代表性的一张, 即 为热点图图1, 如下所示。 


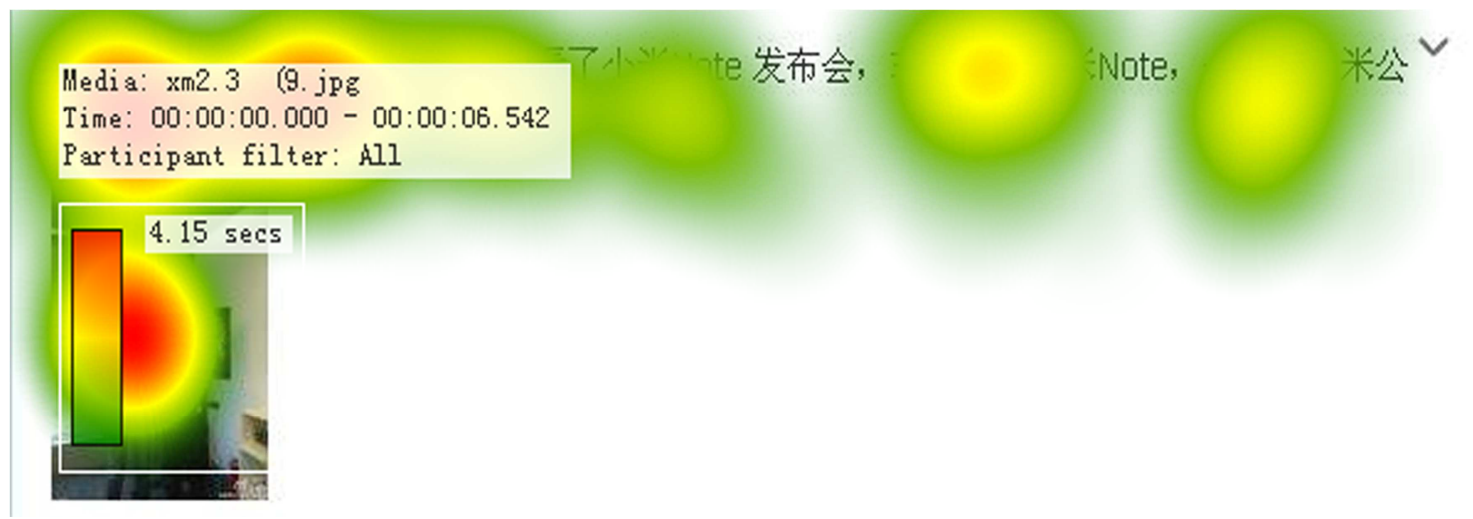

图1 眼动热点图。

图1是在十八张图片中选取了具有代表性的一张, 结 合眼动数据, 可知, 被试者在微博浏览过程中, 普遍对图 片的关注度高于对文字的关注度，微博图片对被试者的关 注度产生显著影响。
将所有被试者对每张图片的平均注视时长汇总起来, 得到表 2 , 并由表 2 做图 2 , 从总体层面观察被试者对任务 导向型互动内容和社会性互动内容的关注差异, 如下所示。

表2 微博图片平均注视时长。

\begin{tabular}{|c|c|c|c|c|}
\hline \multirow{2}{*}{ 图片顺序 } & \multicolumn{4}{|c|}{ 平均注视时长 (秒) } \\
\hline & 第一张图片 & 第二张图片 & 第三张图片 & 总体平均 \\
\hline 1. 企业形象宣传 & 5.52 & 7.36 & 7.17 & 6.68 \\
\hline 2. 手机专业知识 & 4.89 & 6.19 & 4.88 & 5. 32 \\
\hline 3. 企业产品信息 & 4. 45 & 3.44 & 4. 11 & 4. 00 \\
\hline 4. 社会性交流 & 4. 78 & 2.95 & 2.98 & 3.57 \\
\hline 5. 名人娱乐化传播 & 3.65 & 4. 15 & 4. 23 & 4.01 \\
\hline 6. 与粉丝情感沟通 & 2.95 & 4. 26 & 3.13 & 3.45 \\
\hline
\end{tabular}

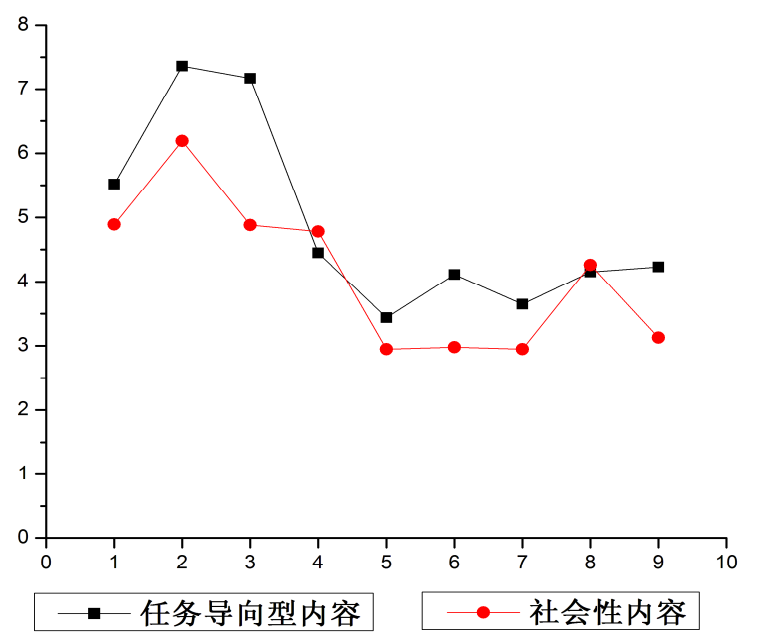

图2 微博图片平均注视时长。

相比较可以看出, 整体而言被试者给予任务导向型互 动内容的注视时间更多，表明相对于社会性互动内容，任 务导向型互动内容更加吸引被试者的注意。但是, 也有可 能因为是第一组图片, 所以被试者给予更多关注。

\section{2. 问卷分析}

表3 信度检验。

\begin{tabular}{ll}
\hline 微博内容 & Cronbach's Alpha \\
\hline 1. 企业形象宣传 & .640 \\
2. 手机专业知识 & .711 \\
3. 企业产品信息 & .780 \\
4. 社会性交流 & .891 \\
5. 名人娱乐化传播 & .902 \\
6. 与粉丝情感沟通 & .863 \\
总体信度 & .904 \\
\hline
\end{tabular}

对每组图片后设置的问题进行信度检验, 采用 Cronbach' s a 值作为信度的判断标准, 测量同一维度下 各测项之间的内部一致性以及量表的整体一致性。 Wortze1 (1979) 主张信度系数介于 $0.70-0.98$ 之间, 视为高 信度, 低于 0.35 则应将其放弃。利用SPSS19.0软件进行分 析, 得到问卷 $\alpha=0.904$, 属于高信度的范畴, 说明问卷设 置具有较高的内部一致性和可靠性, 可以用来开展分析和 研究。

针对被试者对每组图片后设置的四个问题的回答, 分 别进行均值分析, 得到图3, 如下所示。 


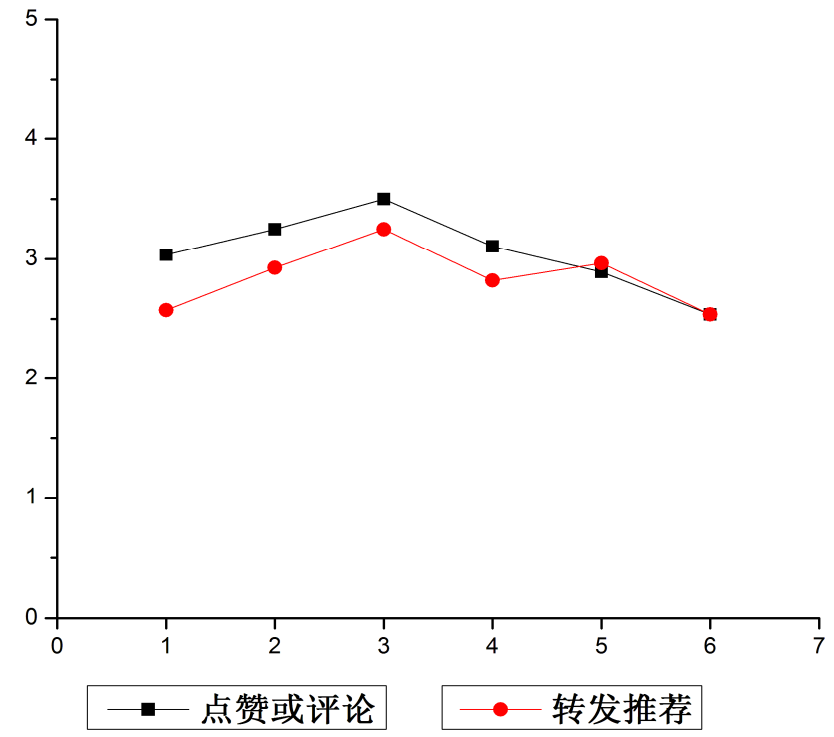

图3 微博满意度均值比较。

从图3可以清楚地看出, 被试者对微博的态度 (是否 愿意评论或点赞, 是否愿意转发推荐) 总体呈山丘状, 在 第三组微博图片, 即产品信息上, 体现出较高的微博满意 度和参与态度, 在第二、四组图片, 即社会性互动内容上 也表现出较高满意度, 而在第一组和第六组图片, 即企业 形象宣传和情感沟通方面, 体现出较低的微博参与意愿。 结合之前预调研的结果, 可以发现, 相比与企业直接相关 的微博内容, 被试者更愿意点赞、评论以及转发社会性互 动内容, 同时, 被试者表现出一定的技术敏感度, 对新发 布的产品兴趣较大。

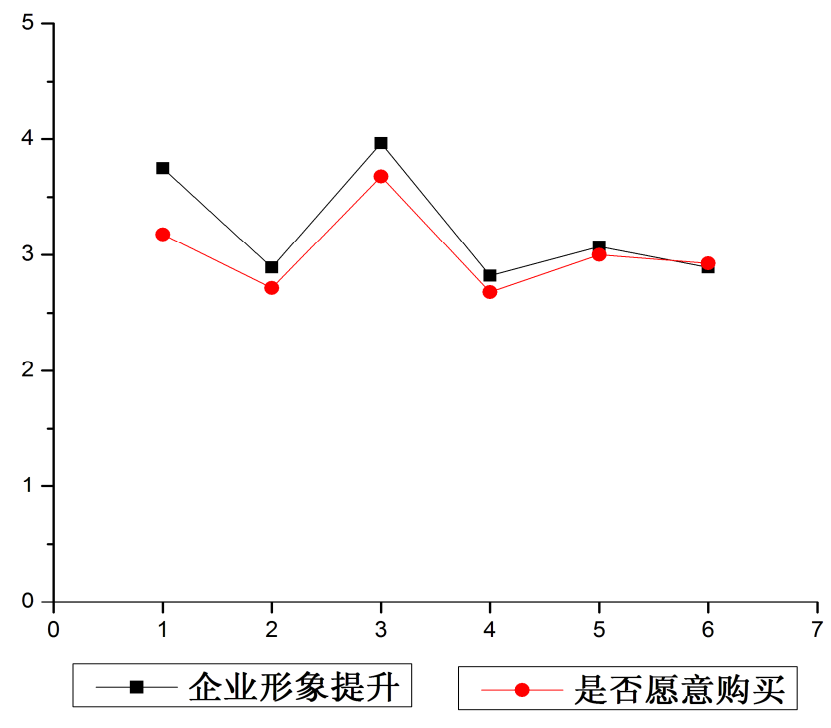

图4 企业满意度均值比较。

针对被试者汶览微博内容之后, 企业形象是否有所提 升, 是否愿意购买企业产品两个问题分别进行均值分析, 得到图4, 如下所示。

图4表明, 被试者通过浏览微博内容, 对小米公司的 满意度 (品牌形象, 购买意愿) 呈现出明显的锯齿状的趋 势，表明第一（企业形象宣传）、三（企业产品信息）、 五 (名人娱乐宣传) 组图片, 即任务导向型互动内容对被 试者企业满意度和购买意愿产生积极影响, 而第二 (手机 专业知识）、四（社会性交流）、六（与粉丝情感沟通） 组图片, 即社会性互动内容, 其得分明显低于任务导向型 互动内容, 且被试者表现出较为消极的态度。

表4 社会性交流Pearson相关性。

\begin{tabular}{lllll}
\hline & Q1是否愿意点赞 & Q2是否愿意转发 & Q3企业形象是否提升 & Q4是否愿意购买 \\
\hline Q1是否愿意点赞 & 1 & $.869^{* * *}$ & $.574^{* *}$ & $.523^{* *}$ \\
Q2是否愿意转发 & $.869^{* *}$ & 1 & $.640^{* *}$ & $.615^{* *}$ \\
Q3企业形象是否提升 & $.574^{* *}$ & $.640^{* *}$ & 1 & $.809^{* *}$ \\
Q4是否愿意购买 & $.523^{* *}$ & $.615^{* *}$ & $.809^{* *}$ & 1 \\
**. 在 .01 水平 (双侧) 上显著相关。 & & & \\
\hline
\end{tabular}

表5 娱乐化传播Pearson相关性。

\begin{tabular}{lllll}
\hline & Q1是否愿意点赞 & Q2是否愿意转发 & Q3企业形象是否提升 & Q4是否愿意购买 \\
\hline Q1是否愿意点赞 & 1 & $.842^{* *}$ & $.649^{* *}$ & $.635^{* *}$ \\
Q2 是否愿意转发 & $.842^{* *}$ & 1 & $.750^{* *}$ & $.527^{* *}$ \\
Q3企业形象是否提升 & $.649^{* *}$ & $.750^{* *}$ & 1 & $.779^{* *}$ \\
Q4是否愿意购买 & $.635^{* *}$ & $.527^{* *}$ & $.779^{* *}$ & 1 \\
**. 在 .01 水平 (双侧) 上显著相关。 & & & \\
\hline
\end{tabular}

分别分析被试者对微博内容态度和对企业态度的相 关性, 六组微博图片均呈现出较为显著的相关关系, 选取 其中第四、五组图片的分析结果, 如表4、表5所示。

由表4、表5可知, 被试者在对微博的态度和对企业态 度之间, 具有显著相关性, 被试者对微博的态度, 会较为 显著地影响其对企业的态度, 被试者对微博内容满意度越 高, 则其对企业满意度和购买意愿也越高。

\section{4. 结论与展望}

\section{1. 结论与建议}

本研究利用眼动实验和问卷调查相结合的方法, 探讨 不同微博内容对被试者态度和购买意愿的影响, 得到以下 几条结论。 
从注视时间和关注点来看, 相比社会性互动内容, 被 试者对任务导向型互动内容给予更多关注。尤其是关于企 业良好形象的微博内容, 比其他微博更能吸引被试者的注 意。由于被试者平时接触到的微博内容, 大多数都属于社 会性内容的范畴, 被试者对社会性互动内容产生审美疲劳, 并不会给予太多的关注。同时, 企业展现出积极向上的企 业形象，以及其他关于企业的正能量信息，更容易吸引被 试者的兴趣。

从被试者对微博内容的满意度来看, 关于企业形象、 情感沟通等任务导向型内容, 被试者微博参与意愿较低, 被试者更愿意评论或者转发社会性互动内容, 比如产生共 鸣的警句, 以及与娱乐名人互动的内容。

被试者对微博内容的态度, 与其对企业的态度显著相 关, 被试者对微博内容满意度越高, 则对企业满意度越高, 购买愿意越大。同时, 任务导向型内容对被试者企业态度 的影响, 要显著高于社会性内容, 被试者在看完任务导向 型互动内容之后, 对企业形象满意度更高, 更愿意购买企 业产品。

结合以上结论对企业微博营销提出一些建议。企业微 博应注重任务导向型内容为主, 社会性互动内容为辅, 利 用二者相互结合的模式来开展微博营销。发布与企业产品 和形象相关的正能量内容, 有效提升企业品牌形象。同时, 注重微博内容的娱乐性, 以文字和图片相结合的方式, 吸 引用户的注意。最后, 调查结果还显示, 企业开展转发或 评论微博, 将有机会获得礼品的活动, 被试者表示出较高 的认同度。企业可以多在微博开展这样的活动, 扩大企业 知名度和影响力。同时，使被试者感受最舒适的企业微博 频率为每天一到三条, 企业也要注意把握好发布微博的频 率, 不要急于求成。

\section{2. 研究不足与展望}

目前在国内管理领域, 利用眼动实验法进行的顾客满 意度研究数量还较少, 本研究在很多方面还需要继续完善 和深入。在实验设计方面, 对图片形式方面的限制还不够 严格, 不能很好控制其他变量对实验结果的影响。问题的 设计也较为简略, 没有设置更多问题, 对结果进行进一步 探讨。同时, 由于采用实验方法收集数据, 导致数据收集
范围较为有限, 样本量不足不仅限制了对眼动实验结果的 精确分析, 也影响了相关问题的研究。

最后, 实验结果的分析还存在值得进一步挖掘的价值。 在将来的研究中, 可以聚焦于被试者特征, 将被试者做出 进一步细分, 有利于企业区分不同顾客类别, 从而有针对 性的开展营销策略。

\section{参考文献}

[1] 刘志明, 刘鲁. 微博网络舆情中的意见领袖识别及分析 [J]. 系统工程, 2011, 29(6): 8-16.

[2] 唐嘉庚. 互动性对B2C环境下信任及购买行为倾向影响研究 [D]. 上海:复旦大学, 2006:23-25.

[3] 陈萍. 从大众传播学角度分析 “微博热” $[\mathrm{J}]$. 大众文艺, 2010 (17) : 134-135.

[4] 查金祥, 王立生. 网络购物顾客满意度影响因素的实证研究 $[J]$. 管理科学, 2006 (1).

[5] 刘丽芳. 微博客的传播特征和传播效果研究 [D]. 浙江杭州: 浙江大学, 2010:30-36.

[6] 魏毅峰, 王传美. B2C 电子商务顾客忠诚度的路径分析 [J]. 商场现代化，2006（28）:56-77.

[7] 董玉. 传统媒体微博营销对消费者品牌态度的影响研究 [D]. 广东广州:暨南大学, 2011:30-34.

[8] 姜宝山, 李雷雷. 微博营销对企业品牌影响的实证研究 [J]. 辽宁工程技术大学学报, 2014 (3).

[9] 田凌云. 提升企业E-忠诚度的网络营销策略 [J]. 中南林业 科技大学学报, 2009 (3) : 104-105.

[10] 杨晓茹. 传播学视域中的微博研究 [J]. 当代传播, $2010(2): 73-74$.

[11] 第34次中国互联网络发展状况统计报告 [R]. 北京: 中国互 联网络信息中心 (CNNIC), 2014. 